\title{
Performance evaluation of multi-ONU customers in ethernet passive optical networks
}

\author{
Oscar J. Ciceri ${ }^{1}$. Carlos A. Astudillo ${ }^{1}$. Nelson L. S. da Fonseca ${ }^{1}$ (1) \\ Received: 31 July 2021 / Accepted: 14 November 2021 / Published online: 26 February 2022 \\ (c) The Author(s), under exclusive licence to Springer Science+Business Media, LLC, part of Springer Nature 2022
}

\begin{abstract}
Resources of Passive Optical Networks (PONs) can be shared by a diverse spectrum of customers such as residential users, business subscribers, mobile network operators, and service providers. A PON customer can rent one or several Optical Network Units (ONUs) from an Infrastructure Service Provider. The ONUs of a customer (multi-ONU customer) can be subject to large spatiotemporal traffic variability, resulting in underutilization of particular ONUs and overutilization of others. The Interleaved Polling with Adaptive Cycle Time with Multi-ONU SLAs support (MOS-IPACT) algorithm has been recently proposed to promote the redistribution of unused bandwidth of underloaded ONUs of a multi-ONU customer. This paper introduces an analytical model to evaluate the duration of idle periods in bandwidth usage, as well as a performance evaluation of the MOS-IPACT algorithm in networks with traditional and multi-ONU customers. Numerical results show that the MOS-IPACT algorithm efficiently distributes the excess bandwidth among the ONUs of multi-ONU customers without compromising the bandwidth availability.
\end{abstract}

Keywords Dynamic bandwidth allocation · Passive optical networks · Quality of service · Multi-tenant · Multi-operator · Multi-customers

\section{Introduction}

Passive Optical Network is a cost-effective broadband access network technology that provides large capacity and low energy consumption. The IEEE-802.3ca and ITU-TG.9804.x standards for PONs provide capacity (up to $50 \mathrm{Gbps}$ ) greater than that of conventional passive optical network (PONs) (i.e., $1 \mathrm{Gbps}$ and $10 \mathrm{Gbps}$ ), which makes their deployment attractive to Infrastructure service Providers (InPs). Besides that, InPs can maximize their revenues by offering various services such as residential access, PON virtualization, mobile backhauling, and fronthauling. Indeed, InPs customers can be end users (e.g., residential subscribers, single or multi-site enterprises), Mobile Network Operators (MNOs),

Nelson L. S. da Fonseca

nfonseca@ic.unicamp.br

Oscar J. Ciceri

oscar@1rc.ic.unicamp.br

Carlos A. Astudillo

castudillo@1rc.ic.unicamp.br

1 State University of Campinas, Campinas, SP, Brazil as well as services providers [1]. Customers can rent one or even several Optical Network Unit (ONU) (multi-ONU customers), as illustrated in Fig. 1. Moreover, Table 1 shows the acronyms used in the paper.

Infrastructure Service Providers can assure bandwidth to PON customers according to Service Level Agreements (SLAs) by employing Dynamic Bandwidth Allocation (DBA) algorithms to distribute the available bandwidth among customers. However, unbalanced traffic load causes ineffective usage of the available bandwidth for multi-ONU customers when traditional DBA algorithms are employed [2]. Moreover, the new social behavior during the COVID19 pandemic yields an increase in the number of remote users, which can result in spatiotemporal traffic variability in different segments of a PON infrastructure. For example, $42 \%$ of the U.S. labor force now works from home full-time [3]; as a result, operators have reported a rise in network utilization of $20-35 \%$ for fixed networks and $10-20 \%$ for mobile networks [4]. In this new scenario, ONUs of multiONU customers (e.g., MNOs) located in the city center and commercial areas tend to underutilize the available bandwidth, while ONUs in the residential areas may starve for bandwidth [5]. 
Table 1 Acronyms

\begin{tabular}{llll}
\hline Acronym & Meaning & Acronym & Meaning \\
\hline AF & Assured forwarding & BE & Best effort \\
CE & Controlled excess & DBA & Dynamic bandwidth allocation \\
DDE & Demand-driven excess & eNB & Evolved NodeB \\
EE & Equally excess & EPON & Ethernet PON \\
FE & Fair excess & InP & Infrastructure service provider \\
MPCP & Multipoint control protocol & MNO & Mobile network operator \\
OLT & Optical line terminal & ONU & Optical network unit \\
PLR & Packet loss ratio & PON & Passive optical network \\
RTT & Round-Trip Time & SLA & Service level agreement \\
TDMA & Time division multiple access & TG & Guard period \\
UE & Uncontrolled excess & QoS & Quality of service \\
VNO & Virtual network operator & - & - \\
\hline
\end{tabular}

Fig. 1 Typically PON deployment with diverse customers

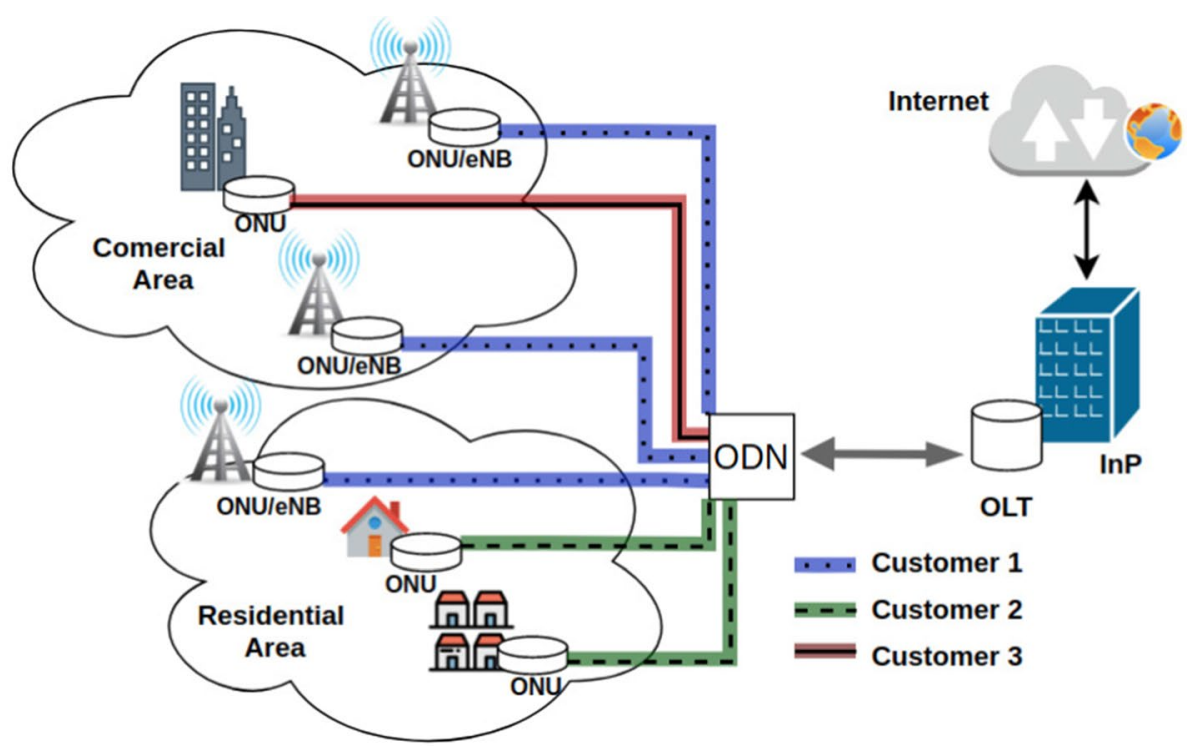

The Interleaved Polling with Adaptive Cycle Time (IPACT) [6] algorithm provides guaranteed bandwidth to individual ONUs, and it is the most employed DBA algorithm in PONs. A variation in IPACT is proposed in [7] to distribute the excess bandwidth among ONUs. The DoublePhase Polling (DPP) algorithm [8] and IPACT with cooperative customers support (CS-IPACT) [9] allow the distribution of the excess bandwidth between two independent subgroups of ONUs and cooperative customers, respectively. The IPACT with Multi-ONU with SLAs Support (MOSIPACT) algorithm [2] allows multi-ONU customers to distribute the excess bandwidth of their underloaded ONUs to their overloaded ONUs, increasing the statistical multiplexing gain. For example, under MOS-IPACT, an MNO multi-ONU customer is able to dynamically redistribute the unused bandwidth from the ONUs serving Evolved NodeBs (eNBs) located in city centers to the ONUs serving eNBs located in suburban areas.
Excess bandwidth distribution policies and scheduling disciplines are crucial to increase the available bandwidth and reduce network access delay. Excess distribution policies determine the redistribution of unused bandwidth of underloaded ONUs to overloaded ones, whereas scheduling disciplines define the events that trigger scheduling decisions. Analysis of the excess policies and scheduling disciplines have been presented in the literature [10-14]. The work in [12] shows the performance of excess distribution policies and their drawbacks. In [10], a mathematical analysis of the bandwidth allocation schemes with excess distribution was provided by using a discrete-time Markov chain model. In [11], the delay produced by various excess distribution policies was evaluated. In [14], an analytical performance analysis on the deterministic delay bound for high and medium-priority traffic was presented. [13] evaluated the performance of offline discipline in Long-Reach PONs. However, no performance evaluation of PONS with 
traditional and multi-ONU customers has been investigated so far, although such analysis is crucial for bandwidth distribution and optimal resource utilization.

The present paper introduces a performance evaluation of the IPACT and MOS-IPACT algorithms employing different excess bandwidth distribution and scheduling disciplines in a scenario with multi-ONU customers. The performance evaluation accounts for packets loss, delay, idle time, and variable load. Simulation results show that MOS-IPACT produces packet loss smaller than that given by the IPACT algorithm for multi-ONU customers under unbalanced traffic. This paper is an extended version of our previously work [15].

The rest of the paper is organized as follows. Section 2 discusses the related work. Section 3 analyzes the idle time produced by the IPACT and MOS-IPACT algorithms. Section 4 details the simulation model, the scenarios employed and discusses results derived via simulations. Finally, Sect. 5 concludes the paper.

\section{Dynamic bandwidth allocation for EPON networks}

Passive Optical Networks (PONs) have no electrical components in the Optical Distribution Network (ODN). The only active elements are the Optical Line Terminator (OLT), located at the Central Office (CO), and Optical Network Units (ONUs), located close to the customers. The distance from the OLT to the ONUs can reach up to $20 \mathrm{~km}$, which is usually sufficient to cover an entire city. Moreover, longreach PONs extend the coverage area of conventional PONs. The ONUs send data to the OLT in dedicated transmission windows using the Multipoint Control Protocol (MPCP) for signaling. The MPCP protocol defines two messages (Gate and Report) for bandwidth allocation. The Gate message is sent on the downlink, from the OLT to the ONUs, to inform the duration and the starting time of its next transmission window. The Report message is sent from the ONUs to the OLT to request bandwidth usage.

Dynamic Bandwidth Allocation (DBA) algorithms define the transmission window in a given cycle according to metrics such as load, delay, guaranteed bandwidth, and excess bandwidth. DBA algorithms for Ethernet PONs (EPONs) employ a grant scheduling discipline, and in some cases, an excess distribution policy. The grant scheduling discipline defines the event that triggers the scheduling of a Gate message. This event can be the arrival of a single Report message (online), the arrival of a Report message from an underloaded ONU (ONU Load Status (OLS)), the arrival of a group of Report messages of two independent subgroups of ONUs [8], or the arrival of Report messages from all ONUs in the PON (offline) [16].

The excess distribution policy defines the distribution of the unused bandwidth $\left(E^{\text {total }}\right)$ of underloaded ONUs $(U)$ to overloaded ONUs $(O)$. The main excess policies are Demand-Driven Excess (DDE), Equally Excess (EE), Weight-based Excess (WE), and Fair Excess (FE) [17]. In the DDE policy, a portion of the unused bandwidth $\left(E_{i}\right)$ is allocated to an overloaded ONU $i$ in proportional to the requested window $\left(W_{i}^{\mathrm{req}}\right)$, according to equation

$E_{i}=\frac{W_{i}^{\mathrm{req}}}{\Sigma_{o \in O} W_{o}^{\mathrm{req}}} \cdot E^{\mathrm{total}}$

In the EE policy, the unused bandwidth is distributed uniformly among the overloaded ONUs, following the equation

$E_{i}=\frac{1}{|O|} \cdot E_{\text {total }}$

In the WE policy, the unused bandwidth is distributed based on the priority weight $\left(\omega_{i}\right)$ of the ONUs, according to the equation

$E_{i}=\frac{\omega_{i}}{\Sigma_{o \in O} \omega_{o}} \cdot E^{\mathrm{total}}$

In the FE policy, the unused bandwidth is proportionally allocated considering the difference between requested window and the maximum transmission window $W_{i}^{\max }$. Thus, $E_{i}$ is calculated by using the equation

$E_{i}=\frac{W_{i}^{\mathrm{req}}-W_{i}^{\max }}{\sum_{o \in O}\left(W_{o}^{\mathrm{req}}-W_{o}^{\max }\right)} \cdot E^{\mathrm{total}}$

These policies are known as uncontrolled excess (UE) policies because the granted windows can be greater than the requested ones. The controlled excess technique (CE) avoids this issue by allocating at most the requested windows. Moreover, the iterative Excess (IE) allocation policy [18] increases the bandwidth utilization of the CE technique by distributing the unused bandwidth.

Several DBA algorithms have been proposed in the literature to guarantee bandwidth provisioning and distribute the excess bandwidth to ONUs in EPON networks. IPACT and MOS-IPACT algorithms provide assured bandwidth to individual ONUs and groups of ONUs, respectively. Moreover, these two algorithms can employ various excess distribution policies and grant scheduling disciplines. However, no performance evaluation of these algorithms has been proposed so far. A comparative analysis is carried out for the first time in this paper. 


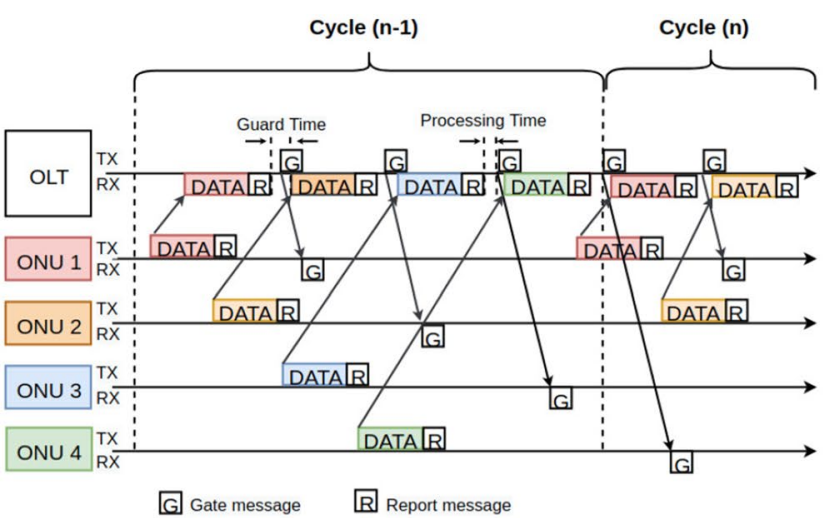

(a) Online discipline

Fig. 2 The IPACT algorithm

\section{Analytical model}

In this section, we derive an analytical model to assess the impact of online and offline disciplines on the idle time in EPON upstream channel when employing the MOS-IPACT and IPACT algorithms.

\subsection{IPACT algorithm}

The IPACT algorithm uses an interleaved mechanism, which reduces bandwidth wastage associated with polling, as shown in Fig. 2a. This algorithm can employ the Limited policy to provide guaranteed bandwidth to ONUs. This policy calculates the granted transmission window $W_{i}^{G}$ for $O N U_{i}$ as the minimum between the requested window $W_{i}^{\text {req }}$ and the allowed maximum window $W_{i}^{\max }$.

The IPACT algorithm can employ an excess distribution policy to improve the statistical multiplexing gain. This policy divides the ONUs into underloaded ONUs and overloaded ONUs at every polling cycle. The former are those requesting at most the maximum transmission window (i.e., $W_{i}^{\mathrm{req}} \leq W_{i}^{\max }$ ), whereas the latter are those with $W_{i}^{\mathrm{req}}>W_{i}^{\mathrm{max}}$. Moreover, the bandwidth of underloaded ONUs is distributed to overloaded ONUs following the arrival of Report messages from all ONUs in the PON (offline), which yields an idle time illustrated in Fig. $2 \mathrm{~b}$.

Let $\mathcal{R}=\left\{r_{1}, r_{2}, \ldots, r_{n}\right\}$ be the set of Round-Trip Time (RTT) values associated with the $n$ ONUs in the PON. For IPACT, the idle time $T^{\text {idle }}=R T T_{\min }+T^{R}+T^{G}$, where $R T T_{\text {min }}$ is the minimum value in $\mathcal{R}$, and $T^{R}$ and $T^{G}$ are the processing times of a Report message and a Gate message, respectively. Moreover, the idle ratio is the idle time divided by the cycle duration. The duration of a cycle includes the transmission windows, guard periods, processing time of Report messages, and idle periods. Note that, the maximum cycle time typically varies between $1 \mathrm{~ms}$ and $10 \mathrm{~ms}$ depending

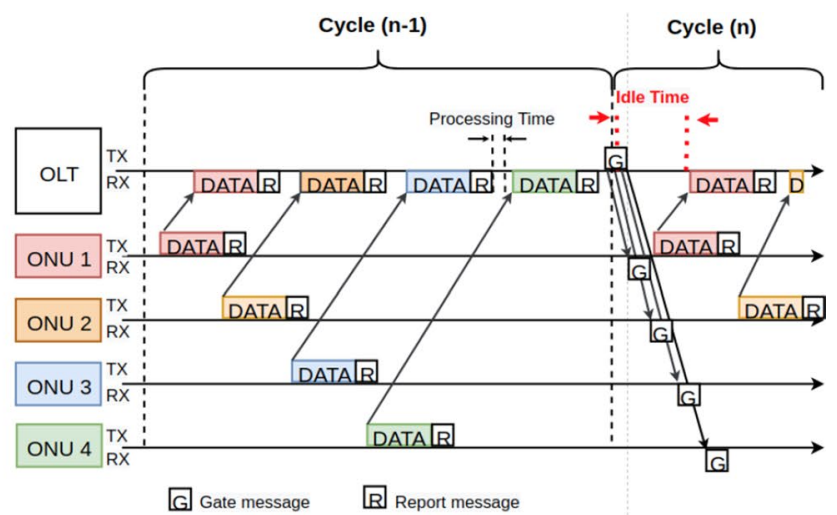

(b) Offline discipline

on the bit rate of the optical link and other requirements. The average idle ratio can be calculated as

$\rho=\frac{1}{C} \sum_{j=1}^{C} \frac{T_{j}^{i d l e}}{\sum_{i=1}^{n}\left(W_{i, j}^{G}+G T+T^{R}\right)+T_{j}^{i d l e}}$,

where $W_{i, j}$ is the transmission windows of $i$-th ONU at $j$-th scheduling cycle, $C$ is the number of scheduling cycles, and $G T$ is the guard period between Report messages at the OLT.

\subsection{MOS-IPACT algorithm}

The MOS-IPACT algorithm combines online and offline grant scheduling. The former is used for scheduling the requests of traditional ONUs, whereas the latter is used for the requests of ONUs of multi-ONU customers. Thus, the interleaved polling in the IPACT algorithm is modified to wait for Report messages from all ONUs of the same multiONU customer before sending the Gate messages to those ONUs, as shown in Fig. 3a. MOS-IPACT employs the Limited policy for traditional ONUs and Limited with excess bandwidth distribution policy for groups of ONUs.

The idle periods produced by MOS-IPACT with offline discipline depend on the transmission windows of traditional ONUs and the $R T T_{\min }$ between the ONUs of the multiONU customers. Figure 3 a illustrates the idle time in the $n$-th cycle for a traditional ONU $\left(O N U_{1}\right)$ and three ONUs $\left(\mathrm{ONU}_{2}, \mathrm{ONU}_{3}\right.$, and $\left.\mathrm{ONU}_{4}\right)$ of a multi-ONU customer in the PON. A variation in the MOS-IPACT algorithm employs the OLS discipline for multi-ONU customers, which reduces the wastage of bandwidth associated with the offline polling, as shown in Fig. $3 b$.

Unlike IPACT, a nonzero idle period in MOS-IPACT offline occurs when the transmission windows of traditional 


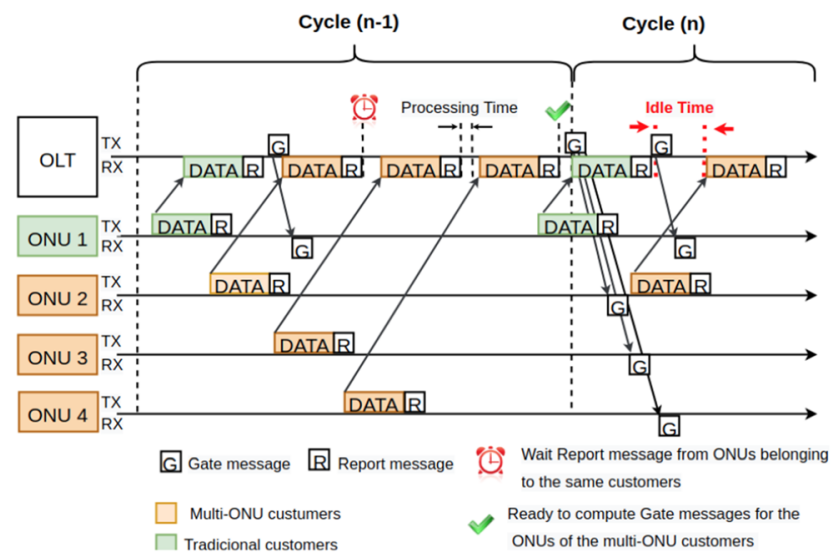

(a) Offline discipline

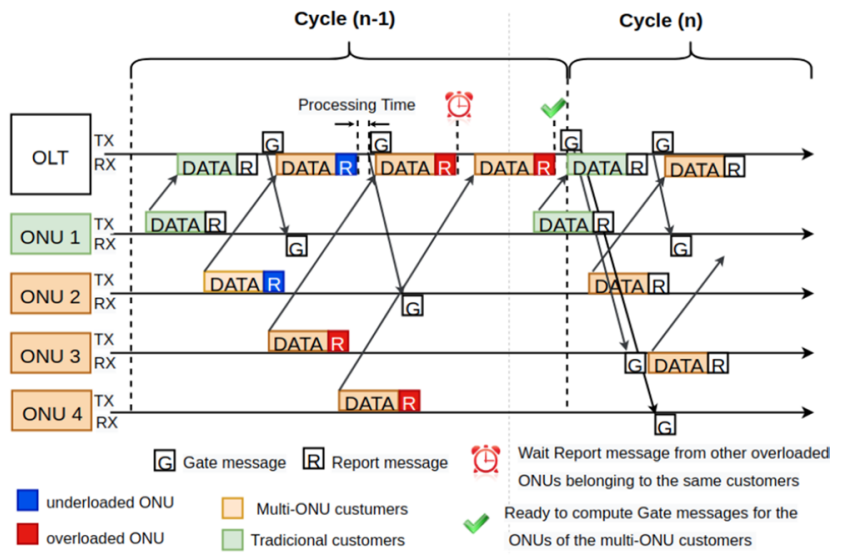

(b) OLS discipline

Fig. 3 The MOS-IPACT algorithm

ONUs are smaller than the minimum RTT of the ONUs in the group $\left(R T T_{\min }\right)$. The idle period produced by MOSIPACT algorithm can be calculated by using

$T^{i d l e}=R T T_{\min }-\sum_{i \in \mathcal{O}_{T}}\left(W_{i, j}^{G}+G T+T^{R}\right)$,

where $O_{T}$ is the set of traditional ONUs. Moreover, the idle ratio depends on the transmission windows of the traditional ONUs

$W^{\text {tra }}=\sum_{i \in \mathcal{O}_{T}}\left(W_{i, j}^{G}+G T+T^{R}\right)$

and the transmission windows of the multi-ONU customer

$W^{m u l}=\sum_{k \in \mathcal{O}_{S}}\left(W_{k, j}^{G}+G T+T^{R}\right)$,

where $O_{S}$ is the set of ONUs of a multi-ONU customer. The average idle ratio in the PON when MOS-IPACT algorithm can be calculated by using the equation

$\rho=\frac{1}{C} \sum_{j=1}^{C} \frac{T_{j}^{\text {idle }}}{W^{\text {tra }}+W^{m u l}+T_{j}^{\text {idle }} .}$

\section{Performance evaluation}

This section assesses the performance of the IPACT and MOS-IPACT DBA algorithms in EPONs with both traditional and multi-ONU customers. We also assess the accuracy of the model proposed in Sect. 3 by comparing its results with those obtained by using simulation. We use the
EPON-Sim simulator (previously validated in $[2,19,20]$ ). The excess bandwidth distribution policies and scheduling disciplines in Table 2 were implemented and extensively validated in the EPON-Sim simulator.

\subsection{Simulation model and setup}

The simulation scenario involves a 10G-EPON network with an OLT serving a set of 32 ONUs $(|\mathcal{O}|=32)$. The traffic in the ONUs is composed of Expedited Forwarding (EF), Assured Forwarding (AF), and Best Effort (BE) representing, respectively, delay-sensitive applications, applications that require bandwidth guarantees, and applications which neither have delay nor bandwidth requirements. The EF, AF and BE types of traffic were modeled as in [2]. The packet length varies uniformly between 64 and 1518 bytes, the maximum cycle length $T_{\max }$ is $1 \mathrm{~ms}$, and the guard period $T G$ is 624ns. Moreover, there is a customer with a set of ONUs $\mathcal{O}_{S} \subset \mathcal{O} ;\left|\mathcal{O}_{S}\right|=N_{\text {group }}$ varying in $\{2,3,4,5,6,7,8\}$. Each ONU

Table 2 Simulated algorithms

\begin{tabular}{llll}
\hline Scheduling & Distribution policy & MOS-IPACT & IPACT \\
\hline Online & Limited & - & ONLINE \\
Offline & Limited with DDE & MOF1 & IPOF1 \\
& Limited with DDE and CE & MOF2 & IPOF2 \\
& Limited with EE & MOF3 & IPOF3 \\
& Limited with FE & MOF4 & IPOF4 \\
& Limited with FE and CE & MOF5 & IPOF5 \\
OLS & Limited with DDE & MOL1 & IPOL1 \\
& Limited with DDE and CE & MOL2 & IPOL2 \\
& Limited with EE & MOL3 & IPOL3 \\
& Limited with FE & MOL4 & IPOL4 \\
& Limited with FE and CE & MOL5 & IPOL5 \\
\hline
\end{tabular}


Table 3 Simulation parameters

\begin{tabular}{ll}
\hline Parameter & Value \\
\hline Optical speed & $10 \mathrm{Gbps}$ \\
Maximum cycle time & $1 \mathrm{~ms}$ \\
Guard band & $624 \mathrm{~ns}$ \\
Distance between OLT and ONUs & {$[10,20] \mathrm{km}$} \\
Propagation delay in fiber & $5 \mu \mathrm{s} / \mathrm{km}$ \\
OLT-ONU RTT & {$[100,200] \mu \mathrm{s}$} \\
ONU buffer size & $10 \mathrm{MB}$ \\
Number of ONUs $(|\mathcal{O}|)$ & 32 \\
Number of ONUs in the group $\left(\left|\mathcal{O}_{S}\right|\right)$ & $2,3,4,5,6,7,8$ \\
Aggregated guaranteed bandwidth in the multi-ONU customer $\left(A_{\mathcal{O}_{S}}\right)$ & $N_{\text {group }} \cdot 300 \mathrm{Mbps}$ \\
Guaranteed bandwidth for the ONUs in the multi-ONU customer $\left(B_{S_{i}}\right)$ & {$[150,450] \mathrm{Mbps}$} \\
Offered load for ONUs in the multi-ONU customer $\left(\lambda_{\mathcal{O}_{S}}\right)$ & {$[0,600] \mathrm{Mbps}$} \\
Number of traditional ONUs & $|\mathcal{O}|-\left|\mathcal{O}_{S}\right|$ \\
Guaranteed bandwidth for traditional ONUs $\left(B_{j}^{T}\right)$ & $(10)$ \\
Offered load in the traditional ONUs $\left(\lambda_{\mathcal{O}_{C}}\right)$ & $B_{j}^{T}$ \\
Intra-ONU scheduler & strict priority \\
\hline
\end{tabular}

in $\mathcal{O}_{S}$ has a guaranteed bandwidth $B_{S k}$, where $O N U_{k} \in \mathcal{O}_{S}$ and $B_{S k}$ vary between $150 \mathrm{Mbps}$ and $450 \mathrm{Mbps}$. Besides that, the mean guaranteed bandwidth in $\mathcal{O}_{S}$ is $300 \mathrm{Mbps}$. The aggregate guaranteed bandwidth in the multi-ONU customer $\left(A_{\mathcal{O}_{S}}\right)$ is $N_{\text {group }} \cdot 300 M b p s$. Moreover, the aggregate offered load $\left(\lambda_{\mathcal{O}_{S}}\right)$ varies from $0 \mathrm{Mbps}$ to $1.0 \cdot A_{\mathcal{O}_{S}} \mathrm{Mbps}^{1}$. The offered load in each $\mathrm{ONU}$ in $\mathcal{O}_{S}$ varies from $0 \mathrm{Mbps}$ to $600 \mathrm{Mbps}$ according to a uniform distribution.

Furthermore, there is a set of traditional ONUs $\mathcal{O}_{T} \subset \mathcal{O}$, with $\mathcal{O}_{T} \cup \mathcal{O}_{S}=\mathcal{O}$ and $\mathcal{O}_{T} \cap \mathcal{O}_{S}=\emptyset$. Each ONU belonging to $\mathcal{O}_{T}$ has a guaranteed bandwidth $B_{i}^{T}$ where $O N U_{i} \in \mathcal{O}_{T}$ and can be calculated by using the equation

$B_{i}^{T}=\left(10 \mathrm{Gbps} \cdot\left(T_{\max }-|\mathcal{O}| \cdot T G\right) / T_{\max }-\left|\mathcal{O}_{S}\right| \cdot 300 \mathrm{Mbps}\right) /\left|\mathcal{O}_{T}\right|$

For example, each $\mathrm{ONU}$ in $\mathcal{O}_{T}$ has a guaranteed bandwidth of $307.64 \mathrm{Mbps}$, when a multi-ONU customer is renting six ONUs in the PON. Moreover, the offered load of traditional ONUs is equal to the guaranteed bandwidth, which is an overloaded condition for ONUs in $\mathcal{O}_{T}$. Table 3 summarizes the parameters used in the simulation.

\subsection{Analysis of wastage of bandwidth}

This section provides relevant insightful comments for efficient bandwidth utilization in EPONs with multi-ONU customers by using the analytical model derived in Sect. 3. In particular, we analyze the average idle ratio produced by the

\footnotetext{
${ }^{1}$ For the sake of clearness and brevity, herein after, $A_{O S}$ is omitted from the offered load values in $\mathcal{O}_{S}$
}

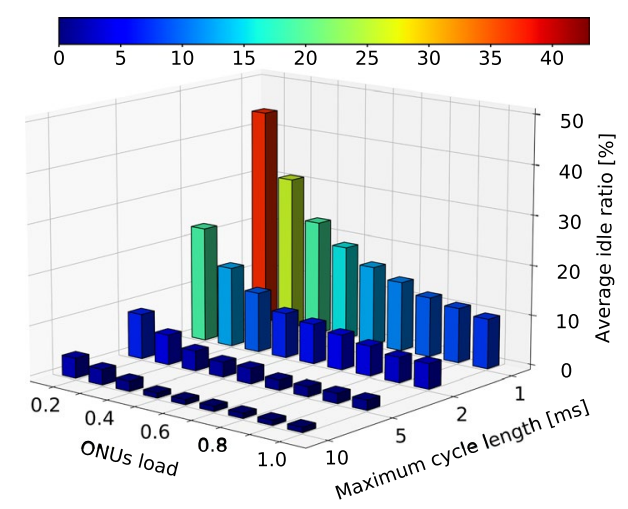

Fig. 4 Average idle ratio produced by IPACT employing an offline discipline

IPACT (employing an offline discipline) and MOS-IPACT algorithms based on (5) and (9), respectively. The DBA processing time was neglected since it is much smaller than the RTT values. Moreover, the duration of the transmission window varies proportionally to the offered ONU load, and all ONUs have the same offered load. For example, an offered load in the traditional ONUs of $0.5\left(\lambda_{\mathcal{O}_{T}}=0.5\right)$ produces a transmission windows of $15 \mu \mathrm{s}\left(W^{\max } \cdot \lambda_{\mathcal{O}_{T}}\right)$.

Figure 4 shows the impact of the maximum cycle duration and the offered load on the average idle ratio when the IPACT algorithm is employed. In this scenario, there are 32 customers (traditional and multi-ONU) with aggregated offered load varying from 0.2 to 1 . The cycle duration varies from 1 to $10 \mathrm{~ms}$. This figure shows that lengthy cycles produce low idle ratios due to the reduction in the proportion between the idle time (which is fixed) and the cycle duration. 


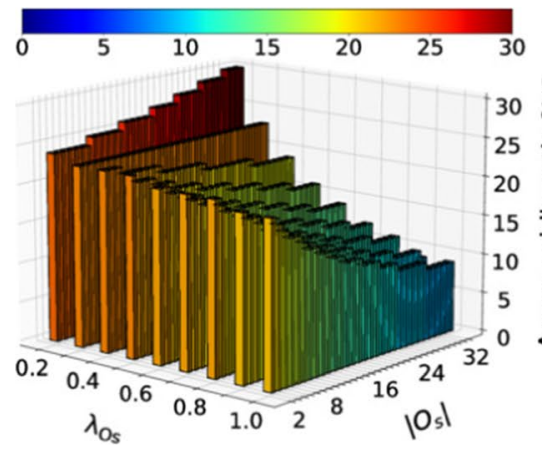

(a) $\lambda_{\mathcal{O}_{T}}=0.3$

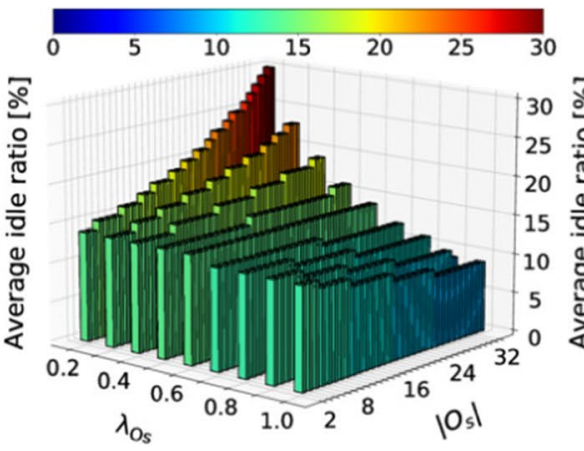

(b) $\lambda_{\mathcal{O}_{T}}=0.6$

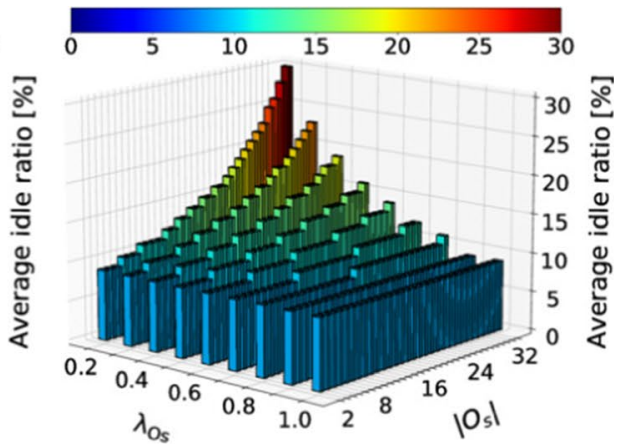

(c) $\lambda_{\mathcal{O}_{T}}=1.0$

Fig. 5 Impact of the offered load and the number of ONUs in the group on the average idle ratio employing IPACT with offline discipline algorithm

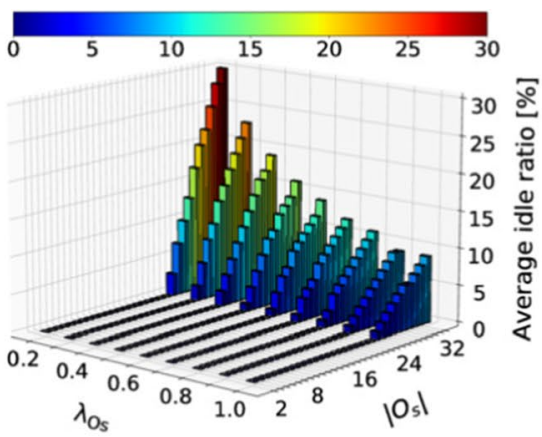

(a) $\lambda_{\mathcal{O}_{T}}=0.3$

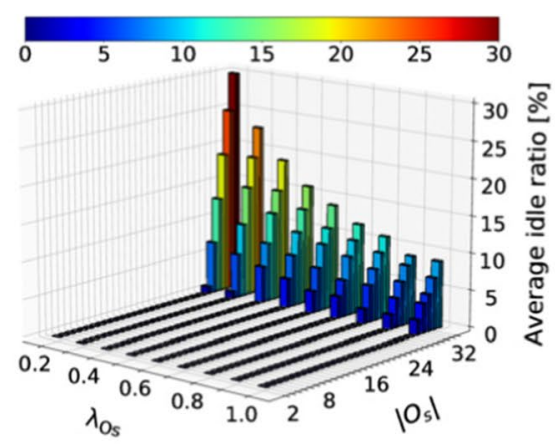

(b) $\lambda_{\mathcal{O}_{T}}=0.6$

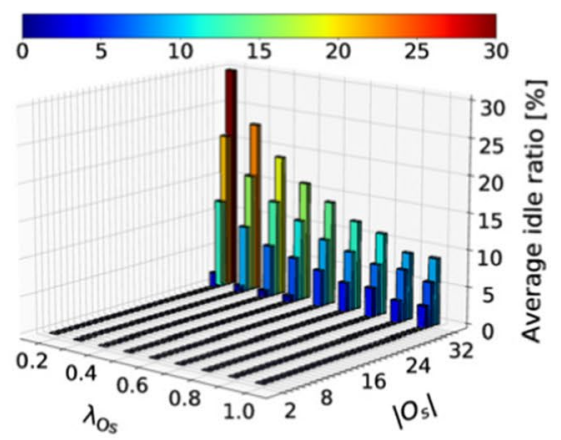

(c) $\lambda_{\mathcal{O}_{T}}=1.0$

Fig. 6 Impact of the offered load and the number of ONUs in the group on the average idle ratio employing MOS-IPACT with online and offline disciplines algorithm

Moreover, short cycles result in idle times from 5\% to $45 \%$, which affects the available bandwidth in PONs.

Figures 5 and 6 show the average idle ratio as a function of the number of ONUs multi-ONU customer $\left(N_{\text {group }}\right)$, the aggregated offered load on traditional customers $\left(\left(\lambda_{\mathcal{O}_{T}}\right)\right.$, and the aggregated offered load on the multi-ONU customers $\left(\lambda_{\mathcal{O}_{S}}\right)$, when the IPACT and MOS-IPACT with offline scheduling algorithms are employed, respectively. In this scenario, the maximum cycle duration is $1 \mathrm{~ms}$.

IPACT offline produces a minimum average idle ratio of roughly $9 \%$, which means that offline scheduling wastes bandwidth regardless of the network load. Moreover, IPACT produces from $15 \%$ to $30 \%$ of average idle ratio when $N_{\text {group }}$ is higher than 23 and $\lambda_{\mathcal{O}_{S}}=0.3$ lower than 0.5. This result evinces that multi-ONU customs renting a large number of ONUs reduce the available bandwidth considerably for all ONUs in the PON when they are underloaded.

Conversely, the MOS-IPACT algorithm produces idle periods only when the multi-ONU customer has more than 22 ONUs (Fig. 6b) and 29 ONUs (Fig. 6c) in underloaded and overloaded conditions, respectively. However, such a number of ONUs belonging to a single customer is improbable to exist. Thus, MOS-IPACT algorithm enables excess bandwidth distribution without affecting the available bandwidth in the PON.

\subsection{Numerical results and discussion}

This section compares the simulation results produced by the MOS-IPACT and IPACT DBA algorithms, using 50 independent replications. The Packet Loss Ratio (PLR), idle time, and number of overloaded ONUs for traditional customers and for multi-ONU customers were analyzed. The IPACT and MOS-IPACT algorithms were compared, employing diverse excess distribution policies and scheduling disciplines (Table 2). The excess policies are FE, $\mathrm{DDE}$, and $\mathrm{EE}$ with/without EC, and the scheduling disciplines are online, offline, and OLS. Moreover, we noted that equivalent results occur when simultaneously varying the load $\left(\lambda_{\mathcal{O}_{s}}\right)$ and the number of ONUs $\left|\mathcal{O}_{S}\right|$ in the multi-ONU customer. For the sake of visualization, the figures presented in this subsection combine algorithms 


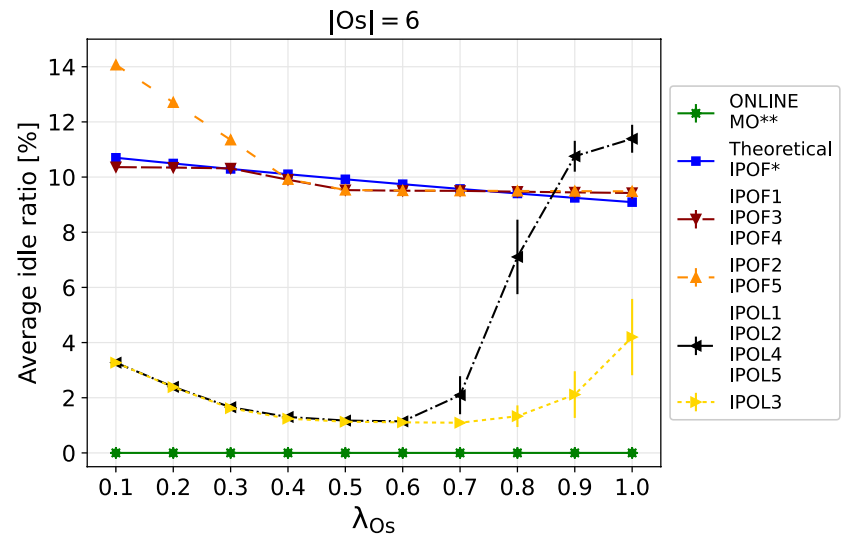

(a) Varying the aggregate load

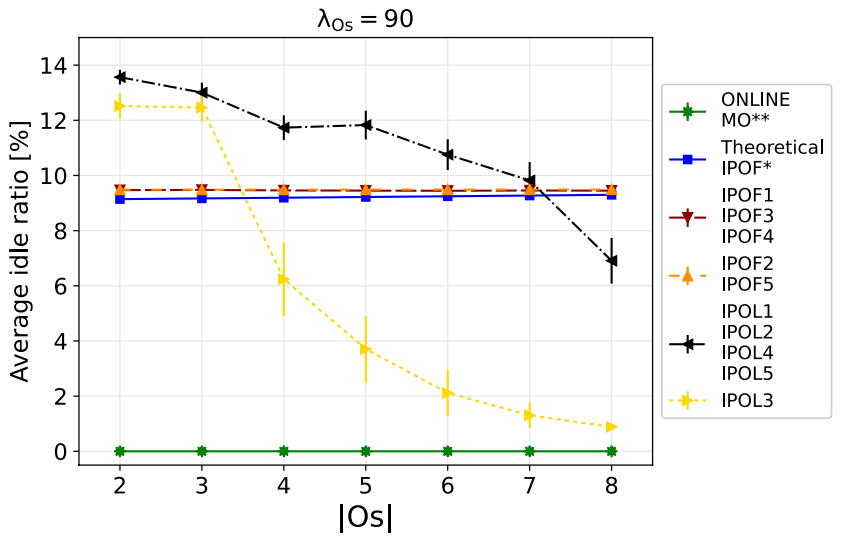

(b) Varying the number of ONUs in the group

Fig. 7 The impact of the offered load and the number of ONUs in the group on the average idle ratio

with similar results by computing a single average value and confidence interval. For example, if the MOL2 and MOF2 configurations in Table 2 produce similar values, we combine their results in a single curve and label it as MO*2 in the figure, where * represents a character at a given position as defined in Table 2 .

\subsubsection{Average idle ratio}

The impact of the offered load and the number of ONUs of multi-ONU customers on the average idle ratio is shown in Fig. 7. This figure shows that the analytical model and the simulation results for IPACT employing an offline scheduling (IPOF*) discipline have similar values. Moreover, IPACT employing online scheduling (IPOL*) discipline produces lower idle values than IPACT using offline scheduling (IPOF*) discipline, which shows that the OLS technique reduces bandwidth wastage. The IPACT OLS with Equally Excess (EE) policy (IPOL3) generates shorter idle times than do other IPACT configurations because the OLT grants extra bandwidth, which increases the use of the optical channel. Thus, IPOL3 gives more data in each transmission to ONUs, reducing the idle time. Moreover, the other OLS schedulers (i.e., IPOL1, IPOL2, IPOL4, and IPOL5) produce shorter idle periods than those given by the IPOF* when the offered load of the multi-ONU customer is less than 0.9 . This cycle reduction occurs because the OLT Report schedules on time the Report messages from underloaded ONUs. Moreover, MOS-IPACT (i.e., MOS-IPACT) and conventional IPACT (i.e., ONLINE) produce no idle time, which is consistent with the analysis in Sect. 3. Thus, MOS-IPACT distributes the guaranteed resources among the ONUs in the group without introducing idle time in the PON.

\subsubsection{Packet loss ratio}

The impact of the aggregated load $\left(\lambda_{\mathrm{Os}}\right)$ and the number of ONUs in group $\left|\mathcal{O}_{S}\right|$ on the packet loss of the multi-ONU customers and traditional customers are shown in Figs. 8 and 9, respectively. The MOS-IPACT algorithm produces PLR values lower than 3\% for multi-ONU customers under overloaded conditions $\left(\lambda_{\mathrm{Os}}=1\right)$ and no packet loss occurs under underload conditions $\left(\lambda_{\mathrm{Os}} \leq 1\right)$ (Fig. 8a). Moreover, there is no packet loss when MOS-IPACT is employed (Fig. 8b) despite the increase in the number of ONUs in the multi-ONU customers. These results show that MOS-IPACT distributes the unused bandwidth effectively among the overloaded ONUs of multi-ONU customers, even under a highly unbalanced load. Furthermore, the scheduling disciplines (i.e., offline or OLS) produce the same PLR for multi-ONU customers when MOS-IPACT is employed. These results are a consequence of both disciplines not wasting bandwidth when $\left|\mathcal{O}_{S}\right|$ is lower than 23. Moreover, IPACT produces packet loss values for the multi-ONU customer up to one order of magnitude greater than those given by MOS-IPACT . Such an outcome is due to the large idle periods produced by the scheduling disciplines when the IPACT algorithm is used. However, the OLS discipline with EE policy (IPOL3) produces lower PLR values than those given by the other IPACT configurations. IPOL 3 produced no packet loss until an offered load of 0.8 in multi-ONU customers. This PLR reduction occurs because the OLT immediately sends Gate messages to the underloaded ONUs when the OLS scheduling discipline is used. Besides that, the OLT grants additional bandwidth when the EE policy is employed, which mitigates bandwidth wastage in multi-ONU customers. The other IPACT configurations produce PLR values higher than $25 \%$ under overloaded conditions since IPACT does not guarantee bandwidth at the level of a group of ONUs. 


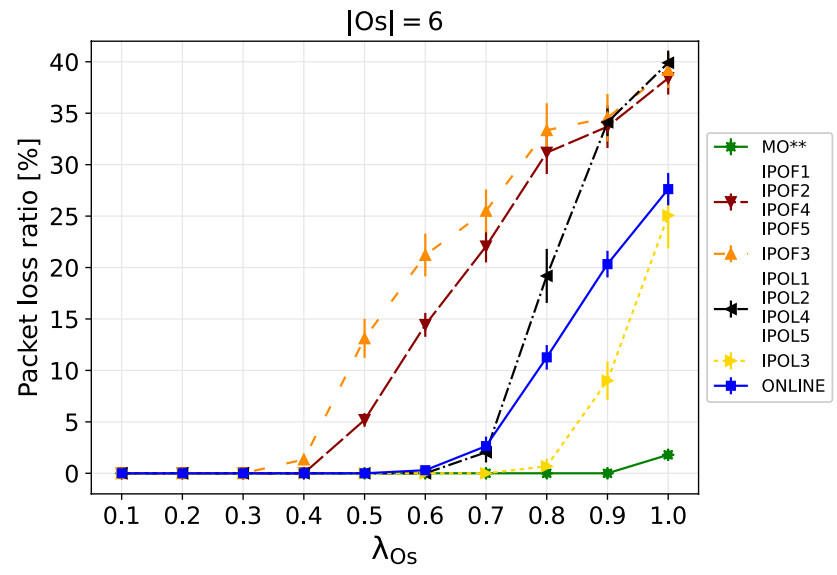

(a) Varying the aggregate load

Fig. 8 Packet loss ratio of the BE traffic in the multi-ONU customer

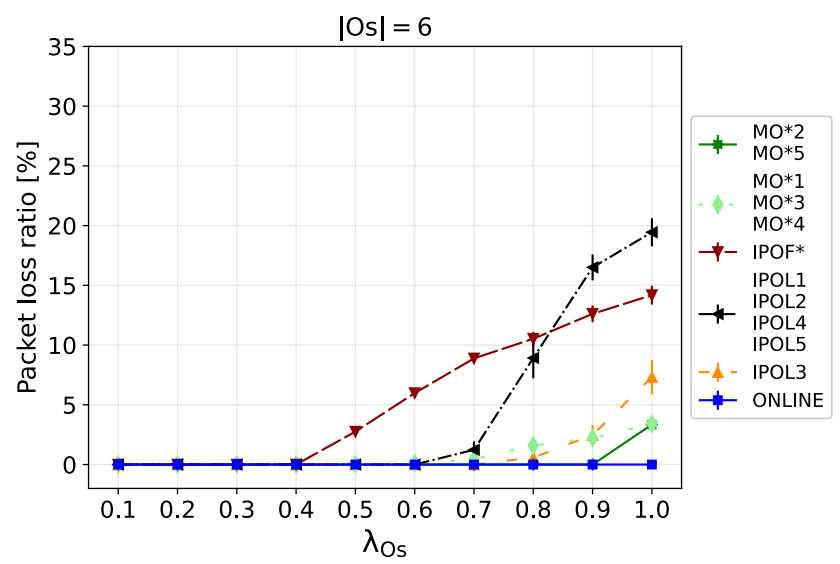

(a) Varying the aggregate load

Fig. 9 Packet loss ratio of the BE traffic in the traditional customers

Hence, overloaded ONUs cannot use the extra bandwidth of the underloaded ONUs.

On the other hand, MOS-IPACT produces PLR values lower than 3\% for traditional customers (Fig. 9a). Moreover, the MOS-IPACT uncontrolled excess (i.e., MO*1, M0*3 and $\mathrm{M} 0 * 4)$ and MOS-IPACT controlled excess (MO*2 and M0*5) produce packet loss under offered loads greater than 0.6 and 0.9 , respectively. MOS-IPACT uncontrolled excess generates PLR values from $2 \%$ to $3 \%$ for each value of $\left|\mathcal{O}_{S}\right|$, while MOS-IPACT controlled excess does not produce packet loss for values of $\left|\mathcal{O}_{S}\right|>4$ (Fig. 9b). The unchecked excess policy generates high packet loss values because the ONUs of the multi-ONU customer receive more bandwidth than necessary, producing bandwidth starvation in traditional customers, even under underloaded conditions. Moreover, conventional IPACT (i.e., ONLINE) produces no BE packet loss for traditional customers. On the other hand, IPACT

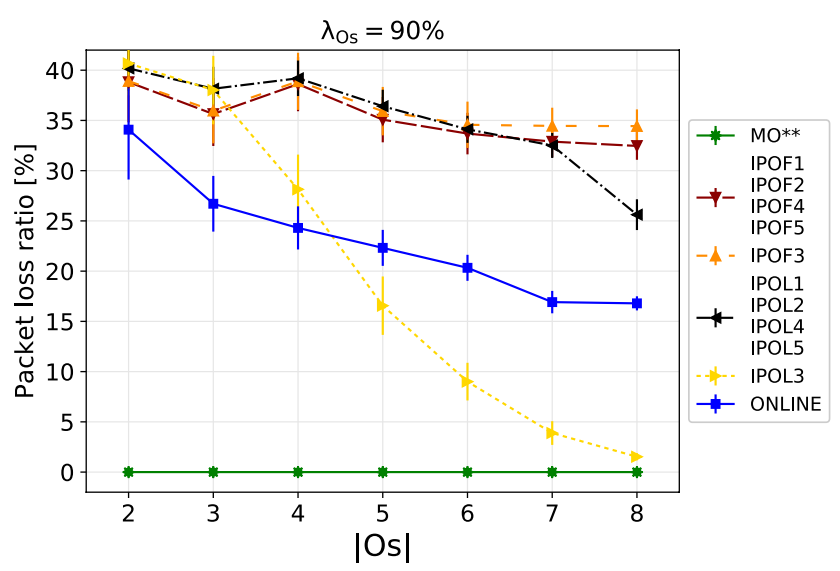

(b) Varying the number of ONUs in the group

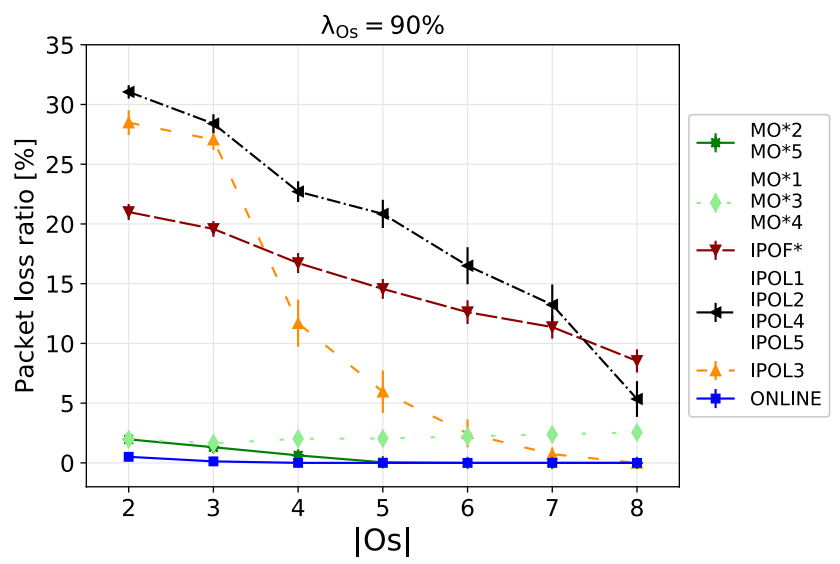

(b) Varying the number of ONUs in the group

employing the offline scheduling produces higher packet loss than that given by IPACT employing the online scheduling and the MOS-IPACT algorithm. These results show that $M O S-I P A C T$ does not affect the guaranteed bandwidth for traditional customers. Moreover, MOS-IPACT reduces the packets loss of the BE traffic of multi-ONU customers than does the IPACT algorithm with excess bandwidth distribution. Thus, MOS-IPACT can redistribute bandwidth without affecting the packet loss of traditional customers sharing a PON with multi-ONU customers.

\subsubsection{Average delay}

The MOS-IPACT and IPACT algorithms produce delay values for the AF traffic of traditional customers lower than $1 \mathrm{~ms}$ because traditional customers have guaranteed bandwidth nearly twice the bandwidth demands of the AF traffic. For 


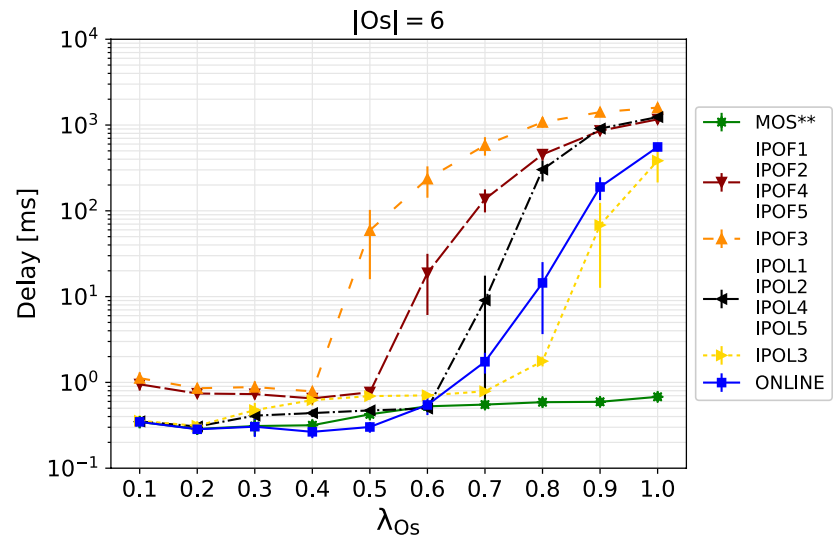

(a) Varying the aggregate load

Fig. 10 Average delay of the AF traffic in the multi-ONU customer

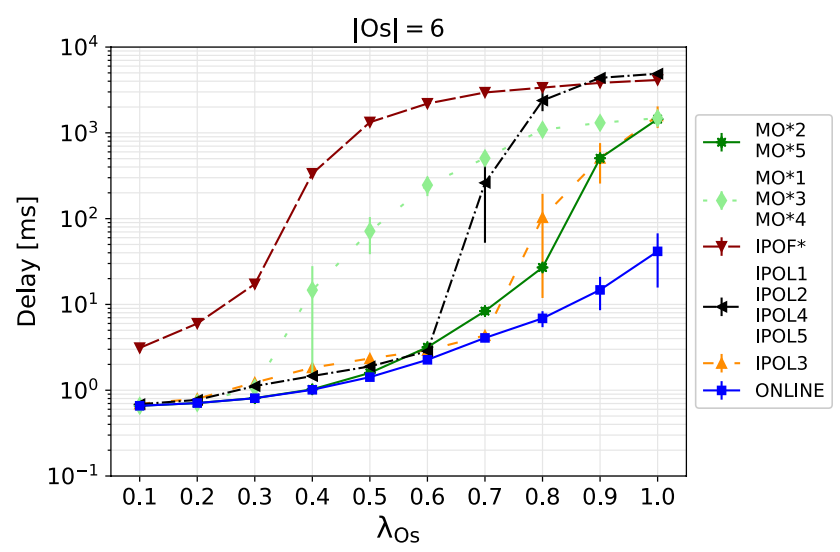

(a) Varying the aggregate load

Fig. 11 Average delay of the BE traffic in the traditional customers

that reason, we assessed the impact of the aggregated load $\left(\lambda_{\mathrm{OS}}\right)$ and number of ONUs in the group $\left|\mathcal{O}_{S}\right|$ on the average delay of the AF traffic in multi-ONU customers (Fig. 10), and the average delay of the BE traffic for traditional customers (Fig. 10).

The MOS-IPACT algorithm produces delay values for the AF traffic of multi-ONU customers lower than $1 \mathrm{~ms}$. These delay values are constant when increasing either the aggregate load (Fig. 10a) or the number of ONUs in the group (Fig. 10b). Thus, the MOS-IPACT algorithm allows multi-ONU customers to support delay-sensitive services for their clients, even under unbalanced traffic conditions. Moreover, the IPACT algorithm generates delay values for the AF traffic of multi-ONU customers two orders of magnitude higher than those generated by MOS-IPACT. IPACT employing an offline discipline generates delay values for the AF traffic one order of magnitude higher than that given by IPACT using an OLS discipline under underloaded conditions (Fig. 10a). This delay increase occurs because the

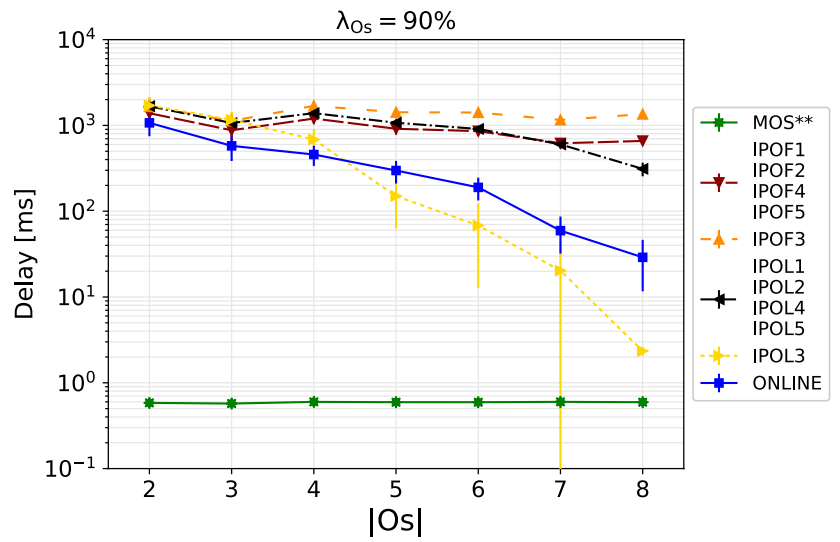

(b) Varying the number of ONUs in the group

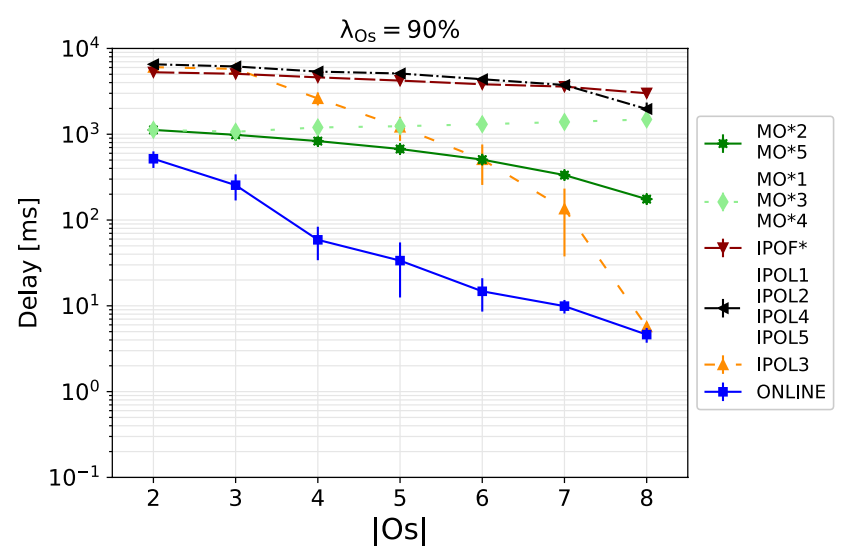

(b) Varying the number of ONUs in the group

OLT cannot redistribute the excess bandwidth of multi-ONU customers among their ONUs when the IPACT is employed. Moreover, the offline discipline increases the idle periods, which reduces the available bandwidth in the PON. Furthermore, the IPOL3 algorithm produces lower delay values for the AF traffic lower than those produced by the other IPACT configurations. In the best case (i.e., $\lambda_{\mathrm{Os}}=1.0$ and $\left|\mathcal{O}_{S}\right|=8$ ), the AF traffic delay value is $2 \mathrm{~ms}$ employing IPOL3 (Fig. 10b).

On the other hand, IPACT employing the online discipline produces delay values for the BE traffic of traditional customers lower than those produced by the other algorithms (Fig. 11). This result is a consequence of the OLT not performing the redistribution of excess bandwidth among the ONUs of multi-ONU customers, which reduces the cycle period. IPACT online gives more data transmission opportunities (extra bandwidth) to traditional customers.

Moreover, MOS-IPACT OLS discipline produces similar delay values to those given by IPACT online until $70 \%$ 


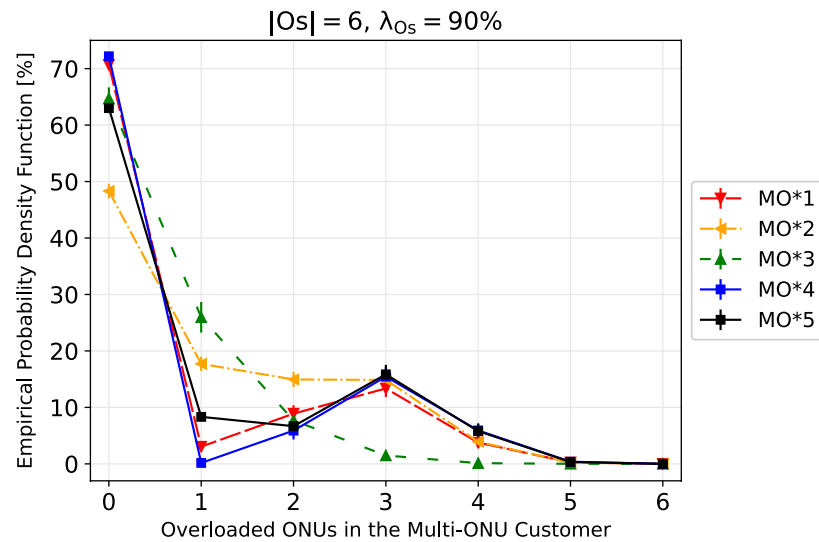

Fig. 12 Probability of overloading in ONUs of the multi-ONU customer

of the aggregated load. However, MOS-IPACT OLS generates delay values one order of magnitude higher than those produced by IPACT online for an aggregated load higher than $80 \%$. Such a negative result occurs because multi-ONU customers use the aggregated guaranteed bandwidth when the MOS-IPACT is used, which increases both the cycle period and the gap between transmission of the traditional customers. Moreover, MOS-IPACT OLS disciplines (MO*2 and MO*5) produce delay values lower than those produced by the MOS-IPACT offline discipline (MO*1, MO*2, and $\mathrm{MO} * 3)$. The OLS discipline reduces the idle period because the OLT sends a Gate message immediately after the arrival of a Report message from an underloaded ONU. MOSIPACT produces no packet loss and delay values lower than $1 \mathrm{~ms}$ for traditional customers; moreover, it produces delays of $1000 \mathrm{~ms}$ for the intermediate (i.e., AF) and low (i.e., BE) priority traffic, respectively. This means that MOS-IPACT effectively isolates highly overloaded traditional customers.

\subsubsection{Number of overloaded ONUs}

The empirical probability of the number of overloaded ONUs of a multi-ONU customer in a cycle is shown in Fig. 12. This customer has six ONUs with an aggregated offered load of 0.9. Even though the excess policies impact on this probability, the same scheduling discipline produces similar results (e.g.,, MOF1 and MOL1). Moreover, the EE policy $(\mathrm{MO} * 3)$ reduces the probability of overloading ONUs of multi-ONU customers. For example, the probability that a multi-ONU customer has three overloaded ONUs is $2 \%$, while this probability is greater than $15 \%$ for the other algorithms. This result is a consequence of the fact that OLT gives more bandwidth than required by the ONUs in the group when the EE policy is employed, which also reduces the bandwidth to the traditional customers. Moreover, the controlled excess policies increase the number of overloaded
ONUs because the OLT grants a transmission window equal to the requested window in the previous cycle, without considering the packets that will arrive at the ONU before the beginning of the transmission.

\section{Conclusion and directions for future research}

This article assessed the performance of various excess distribution policies and scheduling disciplines when both traditional customers and multi-ONU customers coexist in the same EPON networks. The classical IPACT algorithm and the MOS-IPACT algorithm were evaluated.

Results show that the MOS-IPACT algorithm produces efficient distributions of the excess bandwidth among the ONUs without producing idle periods. Moreover, the MOSIPACT algorithm can effectively assure bandwidth for traditional customers and multi-ONU customers coexisting in the same PON. MOS-IPACT employing control excess policies produces more efficient bandwidth utilization and reduces the wastage of bandwidth when compared to the uncontrolled excess policies. Moreover, the equally excess distribution policy allows multi-ONU customers to have a fewer ONUs with bandwidth starvation than do the other excess policies.

Future research directions are envisioned to provide Dynamic Wavelength and Bandwidth Allocation (DWBA) algorithms those allocate the excess bandwidth in time and frequency domain in Time and Wavelength Division Multiplexing (TWDM) EPON networks. Moreover, the marketplace and monetization of the excess bandwidth require a virtual environment, which customers sell/buy the unused bandwidth to other ones. Therefore, we plan to extend the traditional EPONs architecture toward virtualization, in which customers fully manage their excess bandwidth. The requirements for the distribution of excess bandwidth for low-latency applications such as Mobile Frounhauling (MFH) for 5G and 5G Beyond and Ultra Reliable Low Latency Communications (URLLC) are also an interesting problem to be investigated.

Acknowledgements This work was partially sponsored by grant \#15/24494-8, São Paulo Research Foundation (FAPESP), CAPES and CNPq.

Data Availability Statement Data will be made available on reasonable request.

\section{Declarations}

Conflict of interest The authors have no conflicts of interest to declare that are relevant to the content of this article. 


\section{References}

1. Elrasad, A., Afraz, N., Ruffini, M.: Virtual dynamic bandwidth allocation enabling true pon multi-tenancy. In: Optical fiber communication conference, pp. M3I-3. Optical Society of America (2017)

2. Ciceri, O.J., Astudillo, C.A., da Fonseca, N.L.: Dynamic bandwidth allocation with multi-onu customer support for ethernet passive optical networks. In: Proceedings of the 2018 IEEE symposium on computers and communications (ISCC), pp. 00230-00235. IEEE (2018)

3. Jakimowicz, K.: Back to work: How technology can help create a new normal. https://datasmart.ash.harvard.edu/ (2020). Accessed July 2021

4. Bafoutsou, G., Dekker, M.: Telecom security during a pandemic. The European Union Agency for Cybersecurity (ENISA) p. 39 (2020)

5. Heaven, W.: Why the coronavirus lockdown is making the internet stronger than ever. MIT technology review, p. 1. https:// www.technologyreview.com/ (2020). Accessed 10 Sep 2021

6. Kramer, G., Mukherjee, B., Pesavento, G.: Interleaved polling with adaptive cycle time (ipact): a dynamic bandwidth distribution scheme in an optical access network. Photon Netw. Commun. 4(1), 89-107 (2002)

7. Assi, C.M., Ye, Y., Dixit, S., Ali, M.A.: Dynamic bandwidth allocation for quality-of-service over ethernet pons. IEEE J. Sel. Areas Commun. 21(9), 1467-1477 (2003)

8. Choi, S.Y., Lee, S., Lee, T.J., Chung, M.Y., Choo, H.: Doublephase polling algorithm based on partitioned onu subgroups for high utilization in epons. J. Opt. Commun. Netw. 1(5), 484-497 (2009)

9. Ciceri, O.J., Astudillo, C.A., da Fonseca, N.L.: Dba algorithm for cooperative resource sharing among epon customers. In: ICC 2020-2020 IEEE international conference on communications (ICC), pp. 1-6. IEEE (2020)

10. McGarry, M.P., Reisslein, M.: Investigation of the dba algorithm design space for epons. J. Lightwave Technol. 30(14), 2271-2280 (2012)

11. Dutta, S., Das, G., Bhar, C., Chitimalla, D.: Performance analysis of the limited bandwidth allocation scheme with excess distribution. IEEE/OSA J. Opt. Commun. Netw. 11(6), 307-321 (2019)

12. Elrasad, A., Shihada, B.: A practical approach for excess bandwidth distribution for epons. In: OFC 2014, pp. 1-3. IEEE (2014)

13. Mercian, A., McGarry, M.P., Reisslein, M.: Offline and online multi-thread polling in long-reach pons: a critical evaluation. J. Lightwave Technol. 31(12), 2018-2028 (2013)

14. Yin, Y., Poo, G.S.: A hybrid cycle bandwidth allocation scheme with differentiated services support in ethernet passive optical networks. Photon Netw. Commun. 15(3), 263-274 (2008)

15. Ciceri, O.J., Astudillo, C.A., da Fonseca, N.L.: Performance evaluation of ethernet passive optical networks with multi-onu customers. In: Proceedings of the 2020 IEEE Latin-American conference on communications (LATINCOM), pp. 1-6. IEEE (2020)

16. de Melo Pereira, F., Da Fonseca, N.L., Arantes, D.S.: A fair scheduling discipline for ethernet passive optical networks. Comput. Netw. 53(11), 1859-1878 (2009)

17. McGarry, M.P., Reisslein, M., Maier, M.: Ethernet passive optical network architectures and dynamic bandwidth allocation algorithms. IEEE Commun. Surv. Tutor. 10(3), 46-60 (2008)
18. Kim, H., Ha, S., Chiang, M., Kang, D.K., Kim, J.H.: Iterative resource pooling for bandwidth allocation in tdm-pon: algorithm, convergence and experimental evaluation. Photon Netw. Commun. 24(2), 138-150 (2012)

19. Astudillo, C.A., Da Fonseca, N.L.: Standard-compliant qos provisioning scheme for lte/epon integrated networks. IEEE Wirel. Commun. 21(3), 44-51 (2014)

20. Astudillo, C. A., da Fonseca, N. L. S., Borin, J. F.: LTE scheduler for LTE/TDM-EPON integrated networks. IEEE Wirel. Commun. Netw. Conf. (2014). https://doi.org/10.1109/WCNC. 2014.6952396

Publisher's Note Springer Nature remains neutral with regard to jurisdictional claims in published maps and institutional affiliations.

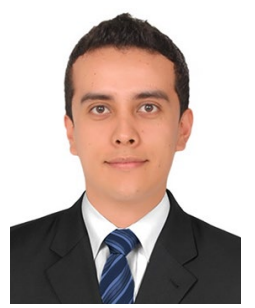

Oscar Ciceri completed his five-year degree in Electronic and Telecommunications Engineering at Universidad del Cauca (UNICAUCA), Colombia, in 2015, and his M.Sc. degree in Computer Science at Universidade Estadual de Campinas (UNICAMP), Brasil, in 2019. $\mathrm{He}$ is Ph.D. student and researcher in the Network Computing Laboratory (LRC) at that university, where he combines research in Passive Optical Networks (PONs) and virtualization. His main research interests are in the area of scheduling and control protocols for broadband access networks.

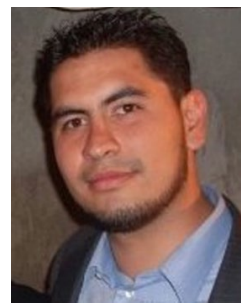

Carlos Astudillo Carlos A Astudillo received his B.Eng. degree in Electronics and Telecommunications Engineering from the University of Cauca, Colombia, and his M.Sc. in Computer Science from the University of Campinas (UNICAMP), Brazil, in 2009 and 2015, respectively. He received 1st Place at the 29th theses and dissertations contest (CTD) of the Brazilian Computer Society (SBC), the 2016 best master's thesis award from the Institute of Computing (IC), UNICAMP, and IEEE best conference paper awards. His areas of interest are machine to machine Communications over cellular networks, machine learning for communications and networking, $5 \mathrm{G}$ optical fronthauling.

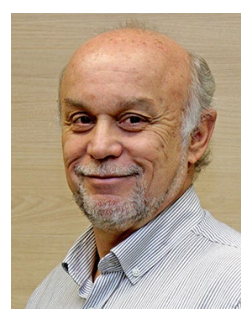

Nelson L. S. da Fonseca obtained his Ph.D. degree from the University of Southern California in 1994. He is a Full Professor at the Institute of Computing, State University of Campinas (UNICAMP). He is Senior editor for the IEEE Communications Magazine and the IEEE Systems Journal. He is also an Associate Editor for Peer-to-Peer Networking and Applications and Computer Networks. He is past EiC of the IEEE Communications Surveys and Tutorials. Nelson served as the IEEE ComSoc VP Publications and VP Technical and Educational Activities. 